\title{
An Automatic Method for Detecting Side Frame Key Using Histogram of Oriented Gradient and Local Binary Pattern
}

\author{
Jinmin Zhang*1, Siming Wang ${ }^{2}$, Yingke Feng ${ }^{1}$ and Tianxi Yang ${ }^{1}$ \\ ${ }^{1}$ School of Mechatronic Engineering, Lanzhou Jiaotong University, Lanzhou, \\ 730070, China \\ ${ }^{2}$ School of Automation and Electrical Engineering, Lanzhou Jiaotong University, \\ Lanzhou, 730070 ,China \\ E-mail: zwls6664@163.com
}

\begin{abstract}
Image processing technology plays an important role in railway fault recognition system. This paper an fault detection method based on histogram of oriented gradient $(H O G)$ and local binary pattern $(L B P)$ for the fault of side frame key(SFK) losing that is one of the freight car typical faults is proposed. The method contained two stages: one is the positioning of the SFK region, and another one is recognition of the fault for the SFK losing. In positioning stage, the application of the improved randomized Hough transform to extract circles on the axle and lines on the side frame get the side frame endpoint. Then, a geometric model is built to calibrate and extract the SFK region according to the geometric relationship among the axle, side frame endpoint and SFK. In the detection stage, the LBP feature histogram and HOG of ROI(region of interest) were statistically respectively, using principal component analysis(PCA) for dimensionality reduction, and the two histogram to constitute a joint histogram. Finally, using support vector machine(SVM) classification, fault detection. Experimental results show that this method the average fault detection rate is $96.15 \%$, and the method has good applicability.
\end{abstract}

Keywords: Side frame key, Randomized Hough transform, Histogram of oriented gradient, Local binary pattern, Support vector machine

\section{Introduction}

In China, with rail transport increasing quickely, the traditional manual testing methods cannot meet the actual testing requirements. Therefore, the ministry of railways is vigorously to promote a safety inspection system-Trouble of moving freight car detection system (TFDS) [1,2]. TFDS uses equipment to shoot fast-moving freight train, image acquisition, and then the use of computer image recognition, thus picking fault $[3,4]$. So, for TFDS, fault recognition is accomplished automatically by computers or man-machines, which greatly enhances the efficiency and reliability of fault detection [5]. Generally, the main fault types include Center-Plate-Bolt losing, Safety Chain dropping, Brake-Shoe-Key losing, Side-Frame-Key losing and so on $[5,6,7,8]$. In these failures, the most common failure is Side-Frame-Key(SFK) losing fault. SFK is bogie on bearing near a small part, generally shaped like a triangular block(Figure 1 (a)). If the SFK losed(Figure 1 (b)), it will result in the derailment of the vehicle and bogie wheel separation, which cannot reduce the extent of damage to the vehicle, but also cause harm to persons [3,4]. Therefore, researches on automatic fault recognition for SFK losing are of great significance to improve the quality and efficiency of the freight train running safety inspection [6].

The automatic fault recognition of freight train is a new application in the field of pattern recognition [6]. As to the losing fault of SFK, some scholars have carried on the exploration and research. Zhu et al proposed a fast potential fault regions locating method 
used in inspecting freight cars [9]. However, this method does not involve SFK losing fault recognition, but simply to SFK region positioning. So, these methods cannot truly achieve SFK losing fault recognition. Sun et al proposed a SFK losing fault recognition algorithm based on shape context [3], but the algorithm efficiency is low. TFDS bogie is divided into two categories: one has no small circle side frame, similar K2 bogie (Figure 1 (a)), the other one is a small circle side frame, similar to K5 bogie (Figure 1 (b)). Sun et al. presented a SFK losing fault automatic recognition method based on geometric model auxiliary positioning, which is simple and reliable [5]. But this method can only discriminate similar K5 bogie in SFK losing fault, and for similar K2 bogie , there is not SFK losing fault recognition.

To address the SFK losing fault recognition problem which are mentioned above. This paper presents a geometric model of auxiliary positioning method based on the improvement, to achieve different type bogie in SFK region positioning, then using histogram of oriented gradient and local binary pattern to realize fault detection.

The remainder of this paper is organized as follows: In Section 2, explaining the design of the fault detection method with all details. In Section 3, the results and data analysis of experiments are discussed. In Section 4, drawing conclusions.

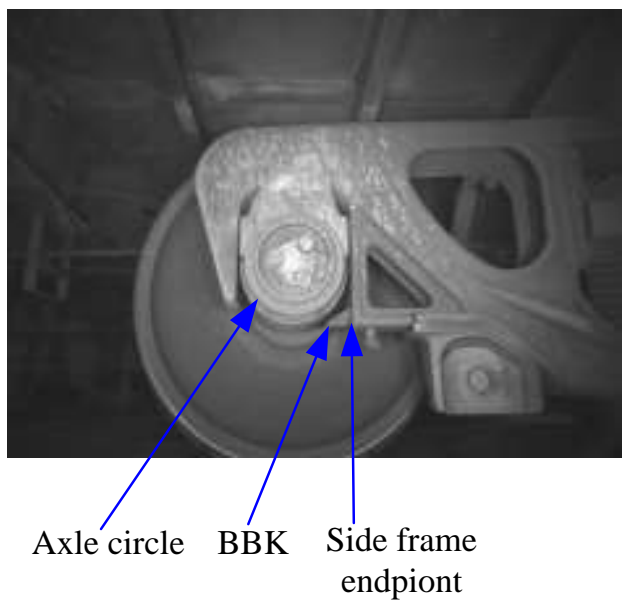

(a) K2 bogie (bogie with BBK)

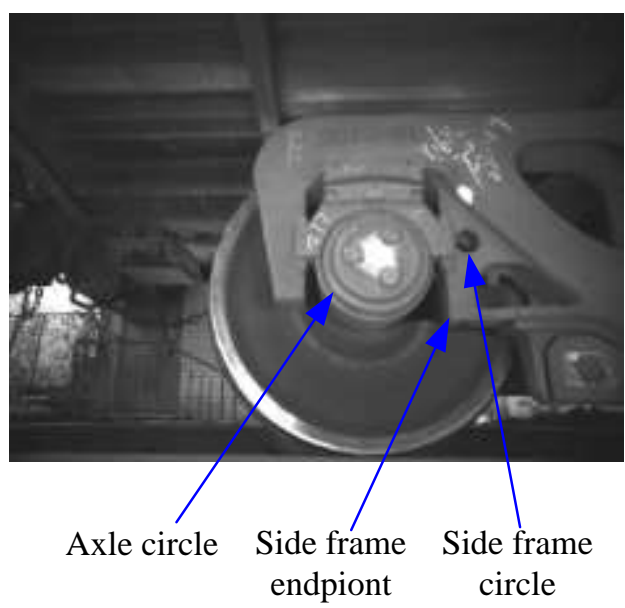

(b) K5 bogie (bogie without BBK)

Figure 1. Bogie with or without SFK and Bogie Type
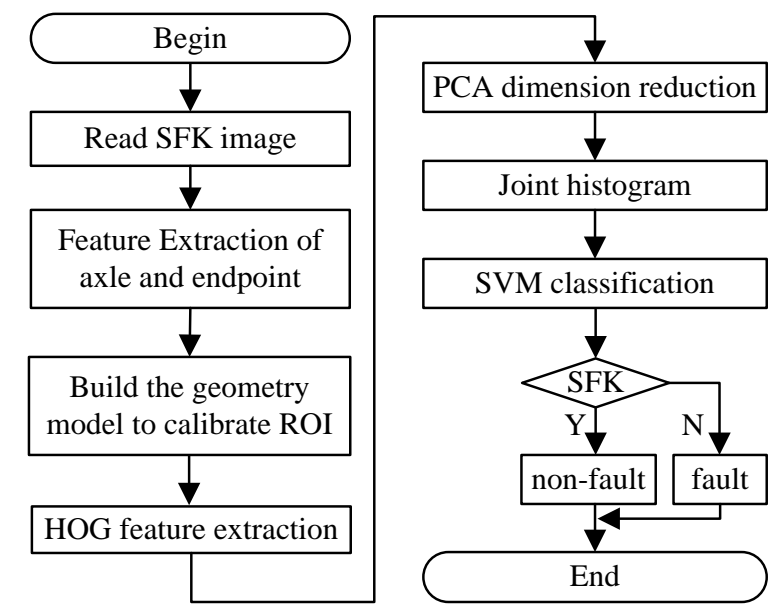
Figure 2. The Overall Framework of Fault Detection Method about SFK
Losing 


\section{The Proposed method}

The SFK appearance is too small, not easy to direct positioning. It uses geometric model to get auxiliary position in this paper and calculate the geometrical are of fault detection.The method consists of three parts: feature extraction of axle and endpoint, the geometric model's building to calibration SFK region, and fault detection. The fault detection the overall framework is shown in Figure 2.

\subsection{Image Filtering}

The image collected by TFDS is shooted in the outdoor, so it is affected by the weather, light and other factors[3,5]. Therefore, the image quality is not pretty good. In this regard, denoising processing is necessary for the image. The common denoising method is image filtering. In order to meet the requirements of the system for image processing speed, this paper uses average filter for image denoising. The average filtering processing result is shown in Figure 3.

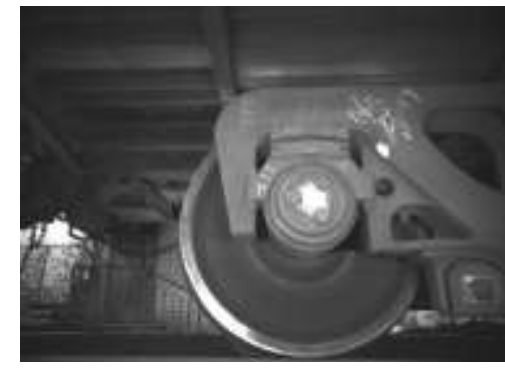

(a) Origin image

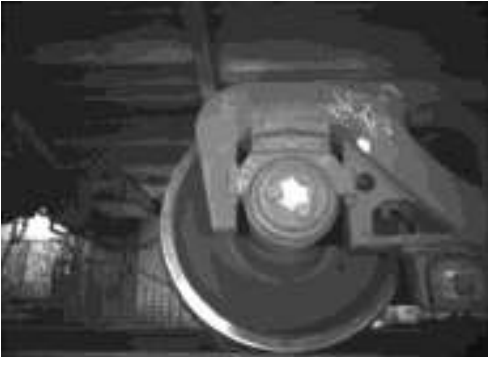

(b) average filtering image

Figure 3. Image Filtering

\subsection{Feature Extraction of Axle and Endpoint}

\subsubsection{Feature Extraction of Axle}

As shown in Figure 1, the bogie axle is circle and the endpoint is the intersection of the side frame two linear. Circle and line detection usually uses Hough transform to get $[10,11]$. In this paper, the situation is circle detection by random Hough transform (RHT), whereas RHT detection efficiency is affected by various factors, where invalid accumulated is the main influence factors [12]. In order to improve the detection efficiency, it is necessary to do the following improvements.

1) Use the region of interest to reduce invalid accumulation

The use of prior knowledge to reduce the detection area can reduce the amount of image data and improve the detection efficiency [13]. The axle and the side frame of the original image from the edge of each of the ${ }^{1 / 6}$ contained in the region, the region is S.

2) Use the improved sampling method to reduce invalid accumulation

Assume that there are $\mathrm{n}$ points in the edge image denoted by $d_{1}, d_{2} \ldots d_{n}$ respectively. For each point, then we collect the number of points located within its $m \times m$ neighborhood area. These numbers are accordingly denoted by $q_{1}, q_{2} \ldots q_{n}$. In this paper, during each sampling, the probability of the point $d_{i}(1 \leq i \leq n)$ being sampled is $Q_{i}$.

$Q_{i}=q_{i}+\frac{1}{\sum_{j=1}^{n}\left(q_{j}+1\right)}$ 
The sampling process can be realized as described below [10].

(1) Define a one-dimension array A whose elements belong to a structure type containing both the horizontal coordinate and vertical coordinate values of the point.

(2) Store the coordinates of all points $d_{i}\left(d_{i x}, d_{i y}\right)(1 \leq i \leq n)$ into A in sequence. There are $q_{i}(1 \leq i \leq n)$ neighborhood points around $d_{i}$, so it stores the coordinates continually for $q_{i}+1$ times. Consequently, the array consists of $\overbrace{\left(d_{1 x}, d_{1 y}\right), \ldots\left(d_{1 x}, d_{1 y}\right)}^{q_{1}+1}, \ldots$ $\overbrace{\left(d_{n x}, d_{n y}\right), \ldots\left(d_{n x}, d_{n y}\right)}^{q_{n}+1}$, where $\overbrace{\left(d_{i x}, d_{i y}\right), \ldots\left(d_{i x}, d_{i y}\right)}^{q_{i}+1}$ (1 $1 \leq i \leq n)$ means that there are $q_{i}+1$ continuous elements $\left(d_{i x}, d_{i y}\right)$.

(3)During the sampling, regard a randomly chosen element in A as the sampled point. If the distance between two points among three sampled points is smaller than the threshold $\varepsilon$, it should sample three points again.

3) Use the gradient to reduce invalid accumulation

Using Sobel operator to find the gradient of the edge points. Assuming that the position relation between current pixel $C_{5}$ and the surrounding eight pixels are as shown in Fig 4.

\begin{tabular}{|l|l|l|}
\hline$C_{1}$ & $C_{2}$ & $C_{3}$ \\
\hline$C_{4}$ & $C_{5}$ & $C_{6}$ \\
\hline$C_{7}$ & $C_{8}$ & $C_{9}$ \\
\hline
\end{tabular}
Figure 4. $C_{5}$ Pixels Relationship between the Current Location and the
Surrounding 8 Pixels

Suppose the gradient of point $C_{5}$ in the $\mathrm{x}$ and y direction component is $G_{x}$ and $G_{y}$. According to the Sobel operato, there are:

$G_{x}=\left(C_{3}+2 C_{6}+C_{9}\right)-\left(C_{1}+2 C_{4}+C_{7}\right)$

$G_{y}=\left(C_{7}+2 C_{8}+C_{9}\right)-\left(C_{1}+2 C_{2}+C_{3}\right)$

The straight line slope of $\mathrm{k}$ along the gradient direction is as follows:

$k=\frac{G_{y}}{G_{x}}$

The linear equation through point $C_{5}\left(x_{0}, y_{0}\right)$ and along the gradient direction is as follows:

$y-y_{0}=k\left(x-x_{0}\right)$

A circle center will be located on the edge of each point on the circumference of a circle of gradient direction, so the intersection point of the gradient direction is the center point. Suppose E, F and G three points are on the same circle, there are three lines of these three gradient directions will intersect at the center. In actual processing process, it needs find intersection of any two lines before calculating distance $d$ from intersection to the 
third line, and then sets the distance threshold value of ${ }^{\varphi}$. If $\mathrm{d}<\varphi$, the three points may be on the same circle, and it's necessary to carry on subsequent calculation and accumulation. Otherwise, it's not necessary and the resampling is in need [14].

4) Use the radius to reduce invalid accumulation

It's known after repeated experiment. axle radius $r \in[120,150]$. Set the maximum radius of $r_{\max }$ and the minimum radius $r_{\min }$ before the program is running [15]. Assuming that the time for the calculation of a parameter is t, then the step calculation time of each radius $r$ value is $2 \mathrm{rt}$. If step range is $\mathrm{R}$, the computation time of each pixel is as follows:

$$
\sum_{r=1}^{R} 2 r t=R(R+1)
$$

When there are $\mathrm{N}$ pixels requirind to be calculated, the required computation time is $\mathrm{NR}(\mathrm{R}+1) t$. So having circular searchin a small range can greatly reduce the calculation time. Using the modified RHT to circle detection can accurately obtain axle circle $\mathrm{O}$.

\subsubsection{Feature Extraction of Endpoint}

Make the following improvements to GHT for straight line detection.

Because the side frame of the two lines is more special. One is approximate to horizontal line and slope Angle is close to $0^{\circ}$, and the other is approximate to vertical line, slope Angle is close to $90^{\circ}$. So the ideas of polar Angle constraint can be used to constrain GHT for straight line detection Angle [16]. Considering the problem of shooting Angle, straight line may tilt. Therefore the set of approximate straight and level Angle in the range is $90^{\circ} \pm 10^{\circ}$, the approximate vertical linear slope Angle within the range is $0^{0} \pm 10^{\circ}$. You can detect in smaller linear slope Angle range to improve the accuracy and efficiency of detection.

Define the gradient direction angle [11] :

$$
\alpha(x, y)=\tan ^{-1}\left(\frac{G_{y}}{G_{x}}\right)
$$

Calculate $\alpha(x, y)$ and evenly divide it into $\mathrm{K}$ intervals. $\alpha_{i}(x, y) \in\left[-\frac{\pi}{2}+\frac{\pi i}{k}, \ldots-\frac{\pi}{2}+\frac{\pi(i+1)}{k}\right], i \in[0, k-1]$. Where $\alpha_{i}$ is a different set of edge pixels. Define statistic $H\left(\alpha_{i}\right)$ as belows.

$H\left(\alpha_{i}\right)=\left\{\begin{array}{l}\sum_{x, y} 1, \alpha(x, y) \in \alpha_{i} \\ 0, \text { other }\end{array}\right.$

Where $H\left(\alpha_{i}\right)$ is the number of pixels of each angle interval. Dividing the edge pixels into different subsets of $D_{i}, i \in[0, k-1]$.Each subset consists of pixels with similar gradient direction. Such voting process requires only a small two-dimensional accumulator to record. A collection of pixels with similar gradient direction of the selected point is likely to be collinear. When dealing with collinear points, the time needed for the voting process and calculation is reduced. That setting a reasonable threshold $\mu$ can filter out inappropriate collection of pixels to eliminate those pixels that have no contribution to straight line detection. Thus, the probability of arbitrary selection of two points on the image of the real straight line will be greatly increased and then the efficiency of detection will improve. 
GHT detected many straight line segments and the line is not easy to obtain a large scale we need, so it needs fitting straight line [17]. Set up linear fitting conditions:

1) Two straight line segment Angle's difference is less than $5^{0}$.

2) Two endpoints of a straight line segment to the other in a straight line segment distance are less than 15 pixels.

In the process of fitting, every two straight line segments participate in the next round of fitting after fitting into a new line segment until there is no longer any two straight line fitting conditions.

So the steps which use GHT to detect linear are as follows:

Step 1: Calculate linear slope Angle k,k is involved in GHT linear detection if it belongs to $90^{\circ} \pm 10^{\circ}$ and $0^{\circ} \pm 10^{\circ}$ range, otherwise it does't participate in GHT linear detection.

Step 2: Calculate $\alpha(x, y)$ and make GHT linear detection.

Step 3: Linear fitting.

Step 4: Calculated the angle between two straight lines. They're two straight lines on the side frame if the angle close to $90^{\circ}$. otherwise, it returns to step 1.

Through using the modified RHT to make line detection, it can accurately obtain two straight side frame, and obtain an endpoint A.

\subsection{Build the Geometry Model to Calibrate ROI}

The geometric model (Figure 5) constructed by literature [5] is based on the geometric relationship between the wheel circle and the side frame circle. The methed of literature [15] is not fit with every conditions such as K2 bogie side frame which locks the key element about the side circle(Figure 1(a)).There is no way to create a geometric model on K2 bogie side frame. Fault identification is identified after positioning, if not set, naturally can not identify similar K2 bogie SFK loss of failure. As that any bogie has wheel and side frame endpoints, this paper proposes a more general geometric model that is depend on geometric models of endpoint and axis geometries by this inherent structural feature, as shown in Figure 6.

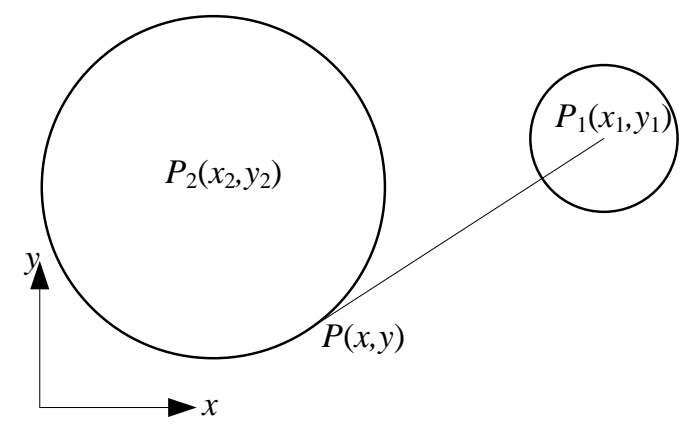

Figure 5. Literature [5] Simplified Geometric Models 


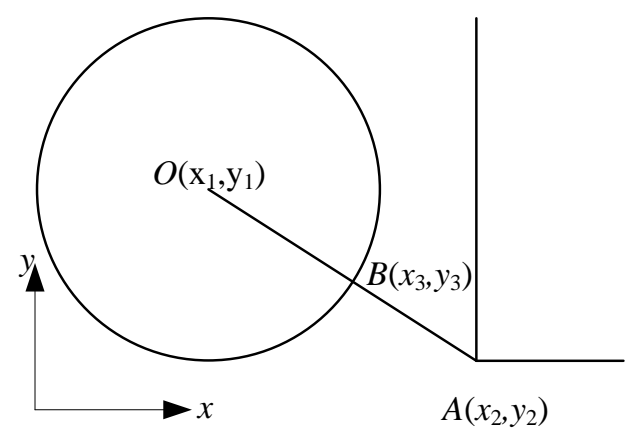

Figure 6. Simplify Geometric Models of the Axle, Endpoint and SFK

Where the center of the circle $\mathrm{O}\left(x_{1}, y_{1}\right)$, radius $\mathrm{R}=\mathrm{r}$ and the coordinates of the endpoints $\mathrm{A}\left(x_{2}, y_{2}\right)$.

In this model, linear OA slope $\mathrm{k}$ can be expressed as:

$k=\frac{y_{2}-y_{1}}{x_{2}-x_{1}}$

General forms of linear equations can be expressed as:

$y=k x+b$

The A coordinates into (10) can be obtained b. So the linear equations of OA can be expressed as:

$y=\frac{y_{2}-y_{1}}{x_{2}-x_{1}} x+\frac{x_{2} y_{1}-x_{1} y_{2}}{x_{2}-x_{1}}$

The equation of circle can be represented as:

$\left(x-x_{1}\right)^{2}+\left(y-y_{1}\right)^{2}=r^{2}$

Simultaneously solving (11) (12), can be obtained only with the circle and linear intersection $\mathrm{B}\left(x_{3}, y_{3}\right)$.

Both A and B points are close to the SFK, so it is possible to consider A, B points as an rectangular diagonal two-point to calibrate ROI. Considering that the bogie SFK has different type, so size is different too. In the process of actual processing, the point $\mathrm{B}$ must be translated of 10 pixels to get $\mathrm{C}$, and $\mathrm{A}, \mathrm{C}$ points as an rectangular diagonal two-point calibrate ROI [5].

\subsection{Fault Detection}

\subsubsection{HOG Feature Extraction}

Histogram of Oriented Gradient (HOG) ,proposed by Dalal and Triggs,is a description operator for pedestrian detection firstly, which is widely used in the field of pattern recognition [18].

HOG feature extraction steps are as follows [18]:

Step1 ROI image grayscale.

Step2 Using Gamma method for ROI image normalization.

$I^{\prime}(x, y)=c I(x, y)^{\gamma}$

Where, $\mathrm{C}, \gamma$ is constant, $\mathrm{I}$ is normalized image.

Step3 Computing the horizontal gradient, vertical gradient of point $(\mathrm{x}, \mathrm{y})$.

$G_{x}(x, y)=I(x+1, y)-I(x-1, y)$ 
$G_{y}(x, y)=I(x, y+1)-I(x, y-1)$

Therefore, the gradient of the point amplitude $G(x, y)$, gradient direction $\alpha(x, y)$ are:

$G(x, y)=\sqrt{G_{x}(x, y)^{2}+G_{y}(x, y)^{2}}$

$\alpha(x, y)=\arctan \frac{G_{y}(x, y)}{G_{x}(x, y)}$

Step4 The image is divided into a number of $S \times S$ Cell with the same size, and censusing the direction gradient histogram of each Cell. Dividing the gradient direction $[-\pi / 2, \pi / 2]$ into $\mathrm{n}$ direction angle region(Bin) averagely, and the gradient direction histogram vectors of each cell are calculated.

Step5 The adjacent Cell $t \times t$ consists of a Block, by the way of Block in image in every direction according to a certain order to move a Cell size, get a Block. and the gradient of Block is the gradient vector from each cell element are connected in series, then the gradient vector for standardization.

Step6 The gradient vectors of each Block are strung together to obtain the HOG features of the ROI, as is shown in Figure 7, which represents extraction process of the HOG feature.

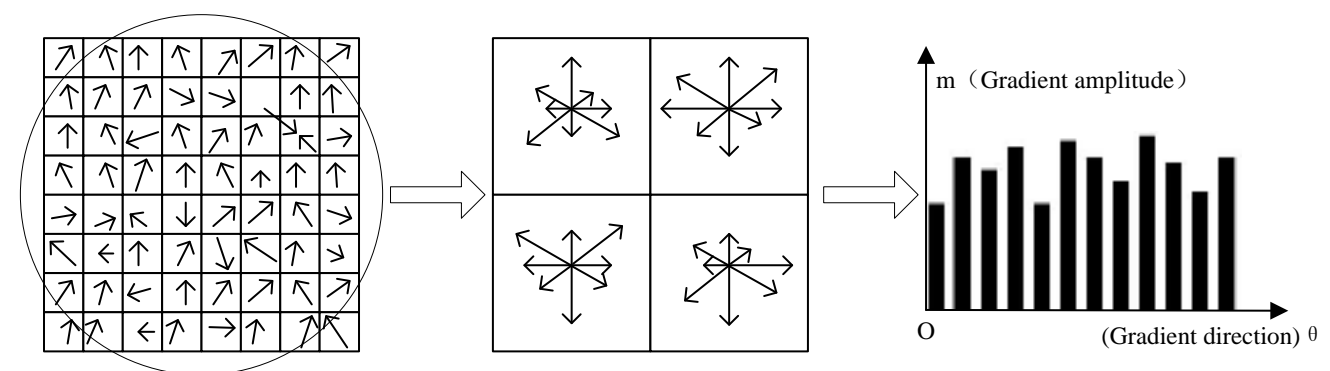

Figure 7. Extraction Process of HOG Feature

HOG histogram of the ROI image can be obtained through the above HOG method, as is shown in Figure8.

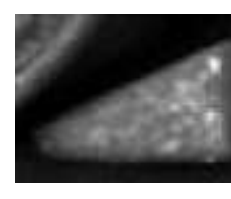

original image

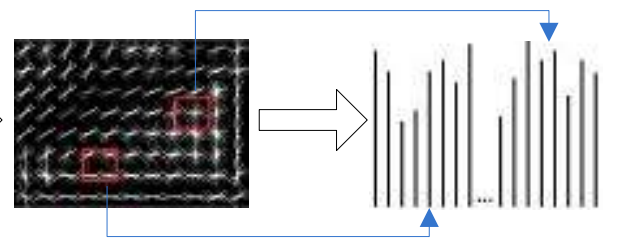

feature of Oriented Gradient

HOG

Figure 8. HOG Histogram

\subsubsection{LBP Feature Extraction}

Local Binary Pattern(LBP), proposed by literature [19], is a operator which expresses local texture features well, therefore, it has a wide range of applications. LBP feature extraction steps are as follows :

Step1 Partitioning detection window to a number of cell with the same size.

Step2 Calculating LBP value. 
Using the LBP value defining center pixel of the window in the $3 \times 3$ block, as is shown in Figure 9.

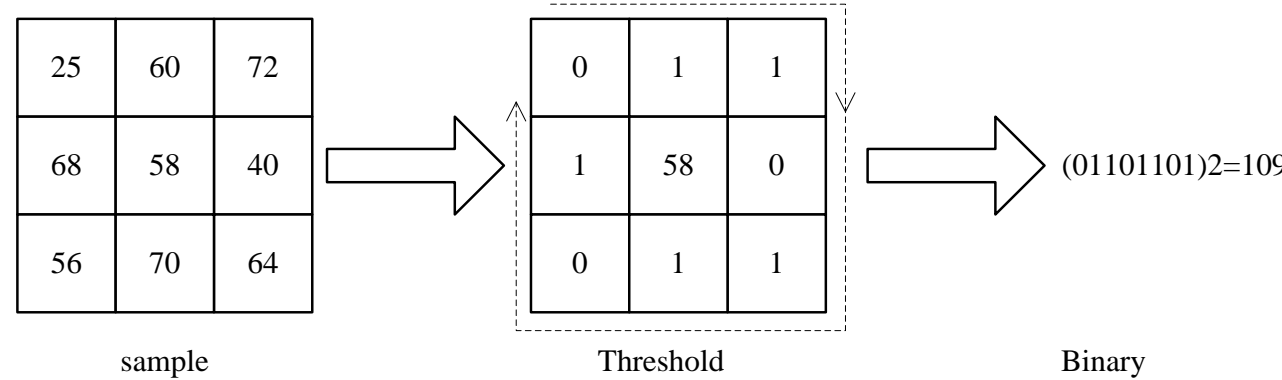

Figure 9. LBP Operator

Step3 Calculating and normalizing the histogram of each cell.

Step4 Connecting the obtained statistical histogram of each cell to be a feature vector to get the LBP texture feature vector of ROI.

The LBP histogram of the ROI image can be obtained through the above LBP method, as is shown in Figure 10.

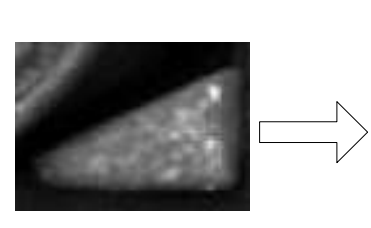

original image

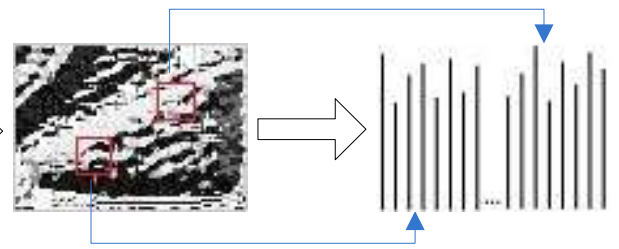

LBP Feature image
LBP Histogram

Figure 10. LBP Histogram

\subsubsection{HOG-LBP Joint Histogram}

Due to the highly calcaulated HOG and LBP feature dimension, it affects the detection speed. PCA method is widely used in dimensionality reduction processing, and the feature dimensionality reduction still owns good description ability [20]. So PCA is used to reduce the dimension of HOG and LBP histogram feature to get the final image low dimensional feature.

Finally, combine the reduced dimension HOG and LBP histogram to form the joint histogram [21], as is shown in Figure11.

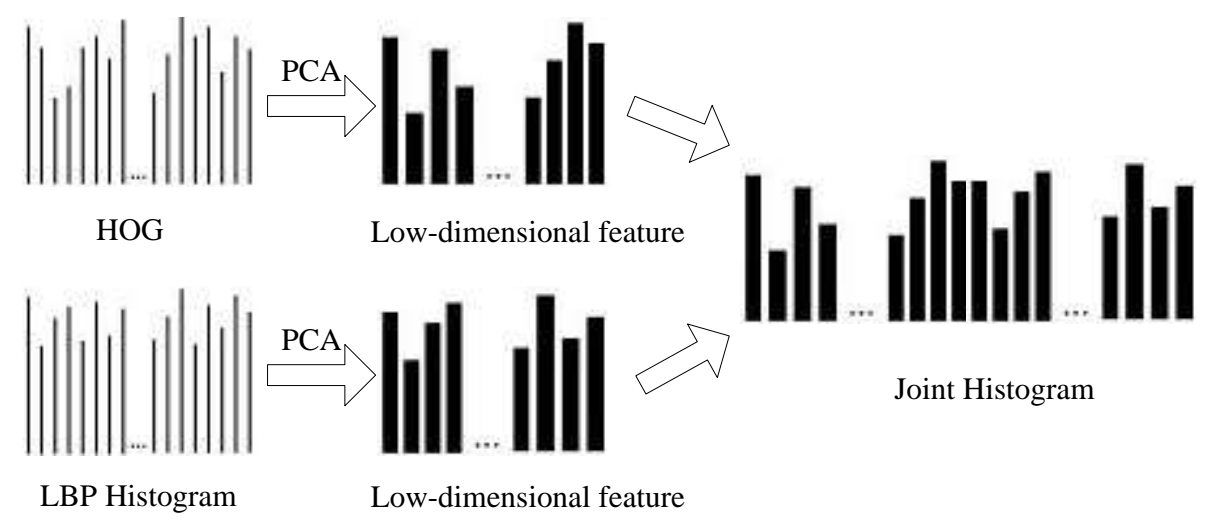

Figure 11. HOG-LBP Joint Histogram 


\subsubsection{SVM Classification}

Using support vector machine(SVM) for classification after extracting ROI image feature. Following is the specific description [22]:

Assumed training sample set $T=\left\{\left(x_{1}, y_{1}\right),\left(x_{2}, y_{2}\right), \ldots,\left(x_{n}, y_{n}\right)\right\}$, There exists a mapping $\varphi\left(x_{i}\right)$, which maps the data $x_{i}$ from the original feature space $\chi$ to the high-dimensional feature space $\mathrm{F}$. The original problem of SVM can be expressed as:

$\min _{w, b, \xi_{i}} \frac{\|w\|^{2}}{2}+C \sum_{i=1}^{n} \xi_{i} \quad\left\{\begin{array}{l}y_{i}\left(w \phi\left(x_{i}\right)+b\right) \geq 1-\xi_{i} \\ \xi_{i} \geq 0 \quad, i=1,2, \ldots, n\end{array}\right.$

Where, $x_{i} \in \chi=R^{2}$ is a sample vector; $y_{i} \in Y=\{+1,-1\}$ is the class number, $\mathrm{N}$ is the number of samples, $\xi_{i}$ is a slack variable, $\|w\|^{2}$ is the Euclidean norm; $\mathrm{C}$ is the cost factor.

The dual problem of primal problem is derived by using Lagrange's function method:

$\max _{\alpha}-\frac{1}{2} \sum_{j=1}^{n} \sum_{i=1}^{n} \alpha_{i} \alpha_{j} y_{i} y_{j} k\left(x_{i}, x_{j}\right)+\sum_{i=1}^{n} \alpha_{i} \quad\left\{\begin{array}{l}\sum_{i=1}^{n} \alpha_{i} y_{i} \\ 0 \leq \alpha_{i} \leq C, i=1,2, \ldots, n\end{array}\right.$

Where, $k\left(x_{i}, x_{j}\right)$ is a kernel function, $\alpha_{i}$ is Lagrange multiplier.

In this paper, the radial kernel function (RBF) effect is good, and the formula is following:

$k\left(x_{i}, x_{j}\right)=\exp \left(-\frac{\left\|x_{i}-x_{j}\right\|^{2}}{2 \sigma^{2}}\right)$

Where, $\sigma$ is the kernel function width.

\section{Experimental Results and Analysis}

In order to verify the proposed method, the SFK obtains an image from the Lanzhou Railway Bureau TFDS system, from which 3000 images were randomly selected as test samples. In Intel Core i7-6700 3.4GHz of CPU, 8GB RAM, In Win7 OS to be tested with MATLAB R2014b, and the test results were analyzed as follows.

\subsection{Method Feasibility Verification}

From the existing samples, two similar K2 bogie SFK images are randomly selected. One is fault image (as sample A), and another one is without fault image (as sample B) to verify the feasibility of the method.

Firstly, the improved randomized Hough transform is used to extract the feature of the axle and endpoint, the results are shown in Figure 12. Figure 12 shows that using the randomly improved Hough transform, can accurately obtain the feature axle and endpoint. According to access to the axle and the endpoint characteristics, using 2.3 the proposed method to built the geometric model, the calibration ROI is shown in Figure 13. Figure 13 shows that the proposed method can accurate calibration ROI. Finally, calculate the HOG-LBP joint histogram of ROI, then using SVM classification to detect SFK is losing or not. So, sample A is the SFK not losing(non-fault status), sample B is the SFK losing (fault status), with the actual situation. It can be concluded that this method can effectively recognize the SFK losing fault. 


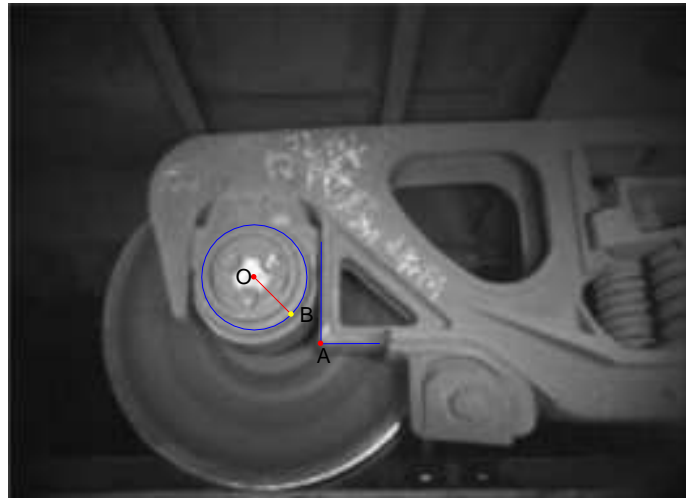

(a) Sample A

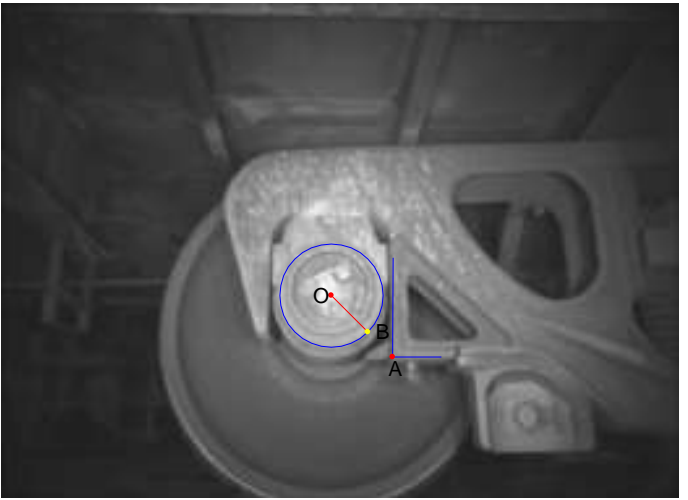

(b) Sample B

Figure 12. Sample $A$ and Sample $B$ the Feature Extraction of Axle and Endpoint

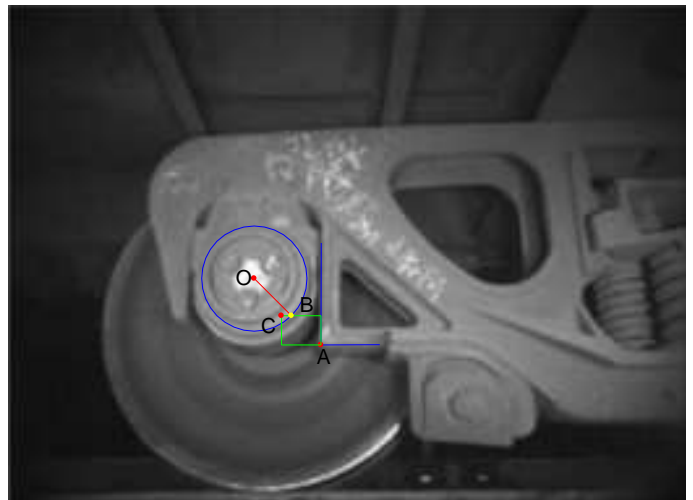

(a) Sample A

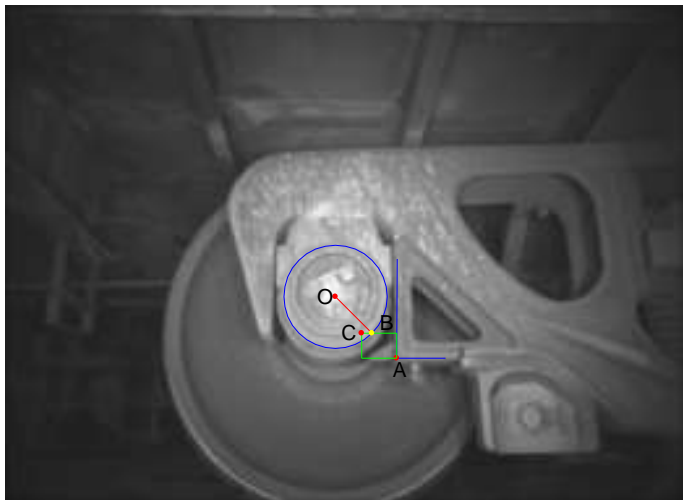

(b) Sample B

Figure 13. Sample A and Sample B the Calibration ROI

\subsection{Method Superiority Verification}

In order to further verify the superiority of the method, some test samples are analyzed. To illustrate the applicability of the method in this paper, the method is used to detect the similar K5 bogie SFK losing fault, the test results as shown in Figure 14. By looking at Figure 14 shows that the proposed method can obtain accurate axle and endpoint features, accurately calibrate SFK region, calculate ratio and recognize fault. Compared with Figure 13 and Figure 14 ,it shows that the method can not only recognize the similar K2 bogie losing fault SFK, but also the similar K5 bogie losing fault SFK, so the method presents good applicability. 


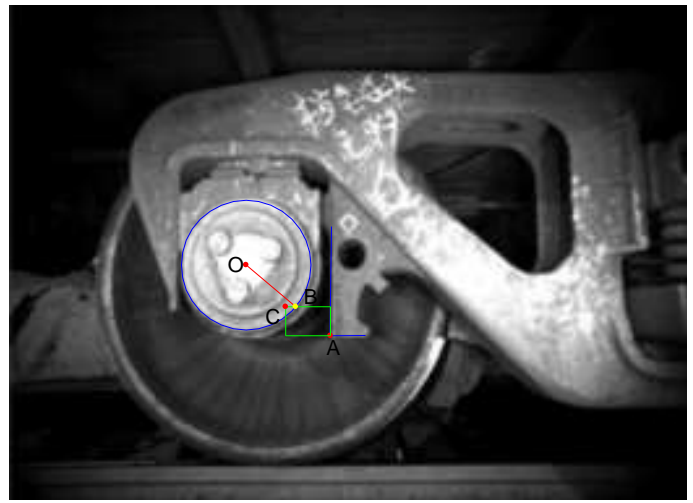

(a) Similar K5 bogie without SFK

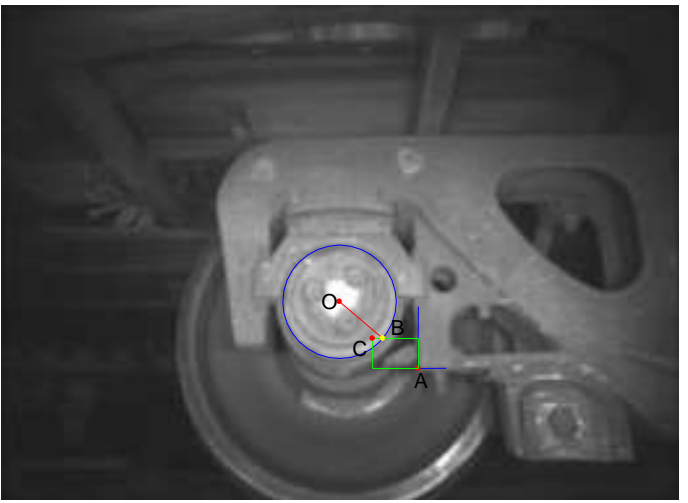

(b) Similar K5 bogie with SFK

Figure 14. This Method Detection Fault about K5 Bogie SFK Losing

In order to illustrate the detection performance of the method, select a certain number of SFK image samples to experiment, test results are as follows.

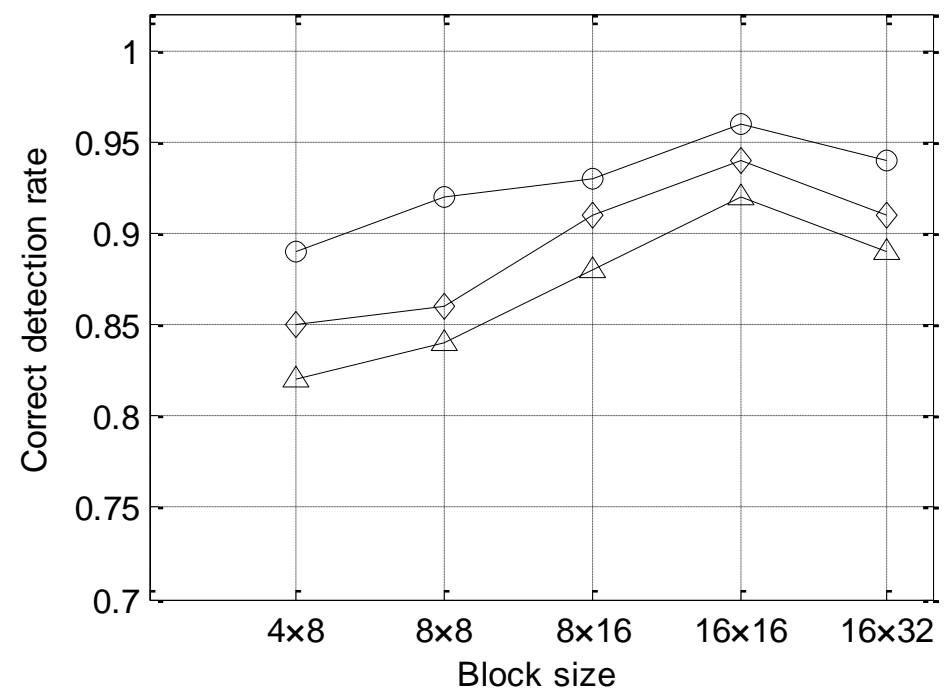

Figure 15. Effect of Block Size

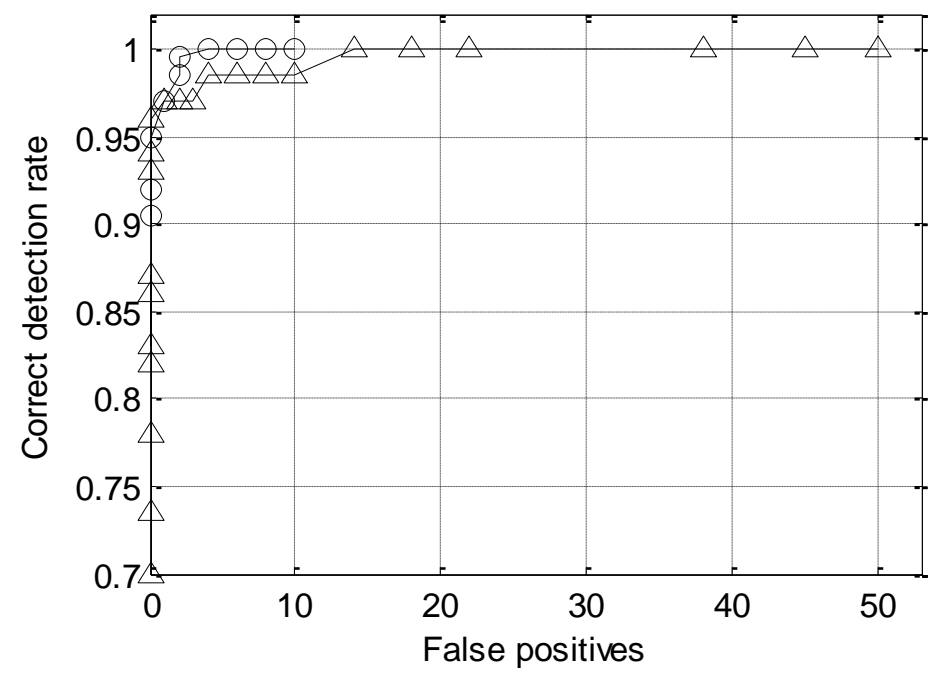

Figure 16. Comparison between HOG-LBP and HOG 
As can be seen from Figure 15, the optimal block size of the 5 block sizes is $16 \times 16$. It can be seen from Figure 16, HOG-LBP when the number of error detection is greater than 10 when the detection rate of $100 \%$, HOG need to detect the number of error is greater than 14 when the detection rate reached $100 \%$. This indicates that in the same case, HOG-LBP is less computationally than traditional HOG. Comparison of HOG and HOG-LBP detection accuracy shows that HOG-LBP detection accuracy is higher. Therefore, HOG-LBP has better detection performance than HOG.

In order to illustrate the accuracy and real-time performance of the method, a certain number of SFK images are select from the existing samples. According to the type of bogie ,two small samples are made. Meanwhile, sample 1 is similar to the similar K2 bogie SFK image, and sample 2 is similar to the similar K5 bogie SFK image. Sample 1 and sample 2 each of 1500 images. Experiments were carried out using different methods, the results are shown in Table 1 Table 2.

Table 1. For the Sample 1 using Comparison of Fault Detection Performance of Different Methods

\begin{tabular}{cccccc}
\hline Detection method & $\begin{array}{c}\text { Normal } \\
\text { image }\end{array}$ & $\begin{array}{c}\text { Fault } \\
\text { image }\end{array}$ & $\begin{array}{c}\text { Number of } \\
\text { detected }\end{array}$ & $\begin{array}{c}\text { detection } \\
\text { rate }(\%)\end{array}$ & $\begin{array}{c}\text { detection } \\
\text { time }(\mathrm{s})\end{array}$ \\
\hline Method of literature [3] & 1200 & 300 & 272 & 90.67 & 2.28 \\
Method of literature [5] & 1200 & 300 & 286 & 95.33 & 0.55 \\
Proposed method & 1200 & 300 & 200 & 96.01 & 0.57 \\
\hline
\end{tabular}

Table 2. For the Sample 2 using Comparison of Fault Detection Performance of Different Methods

\begin{tabular}{cccccc}
\hline Detection method & $\begin{array}{c}\text { Normal } \\
\text { image }\end{array}$ & $\begin{array}{c}\text { Fault } \\
\text { image }\end{array}$ & $\begin{array}{c}\text { Number of } \\
\text { detected }\end{array}$ & $\begin{array}{c}\text { detection } \\
\text { rate }(\%)\end{array}$ & $\begin{array}{c}\text { detection } \\
\text { time(s) }\end{array}$ \\
\hline Method of literature [3] & 1150 & 350 & 318 & 90.86 & 2.32 \\
Method of literature [5] & 1150 & 350 & 0 & 0 & - \\
Proposed method & 1150 & 350 & 337 & 96.29 & 0.55 \\
\hline
\end{tabular}

As shown in Table 1 and Table 2, comparing the three methods of detection number, they are presented as follows: the method of literature [5] can only recognize similar K5 bogie losing fault SFK, and the SFK for similar K2 bogie losing fault cannot recognize. Although the method of literature [3] can realize the two type bogie SFK losing fault detection, it needs to select the corresponding template matching for different types of bogie. But this method does not need to consider the bogie type of case to recognize SFK losing fault, which shows the applicability of this method once again.

As is shown in Table 1 and Table 2, Comparing the three methods of average detection rate: in the method of literature [3], average detection rate is lowest and accuracy is the worst. In the method of literature [5] the average detection rate is higher, but the applicability of binding method, still cannot satisfy the actual testing requirements. The method in this paper, the average detection rate is highest-96.15\%. The method owes good applicability, satisfying the requirement of the actual test.

As shown in Table 1 and Table 2, Comparing the three methods of average detection time: detection time of the method of literature [3] is longest, but real-time worst. In the method of literature [5] average detection time is shortest, but the applicability of binding method still cannot satisfy the real-time detection of the TFDS requirements. The method average detection time is $0.56 \mathrm{~s}$, which also meets the requirements of the TFDS system of real-time detection. Under the condition of applicability good guarantee method, the method can also has good detection accuracy and real-time performance, and some practical value. 


\section{Conclusion}

Based on HOG-LBP method for fault detection of SFK losing is proposed. This method not only can achieve different types of bogie ROI fast and accurate positioning, fault detection. In order to improve the detection efficiency of RHT, the use of region of interest ,sampling methods, gradient, radius and gradient angle to reduce the invalid accumulation. Firstly, the original image is filtered. Then, using the improved RHT detection axle and axle and endpoint. According to the establishment of a geometric model calibration of ROI. Finally, calculate the HOG-LBP joint histogram of ROI, then using SVM classification to detect SFK is losing or not. Using the actual image fault test. Experimental results demonstrate that this method with high accuracy and strong robustness the performance is good. The average accuracy rate reached $96.15 \%$, the average time of fault identification for an image is $0.56 \mathrm{~s}$, it can well meet the actual inspection of freight train operation safety requirements. In this paper, we find that the general method of fault identification provides a way of thinking.

\section{Acknowledgments}

This work is supported by the National Natural Science Foundation of China (61263004).

\section{References}

[1] F.Q. Zhou, R. Zou, H. Gao, "Dust collector localization in trouble of moving freight car detection system", Zhejiang Univ-Sci C (Comput \& Electron), vol. 14, no. 2, (2013), pp. 98-106.

[2] R. Zou, Z.Y. Xu, J.Y. Li, F.Q. Zhou, "Real-time monitoring of brake shoe keys in freight cars", Frontiers of Information Technology \& Electronic Engineering,vol. 16, no. 3, (2015), pp. 191-204.

[3] G.D. Sun, W. Xu, Y.Q. Liang, D.X. Zhao, "Image recognition algorithm for side frame key of train based on shape context", Journal of Railway Science and Engineering, vol. 11, no. 6, (2014), pp. 127-131.

[4] H.J. Zhang, P. He, X.D. Yang, "Fault Detection of Train Center Plate Bolts Loss Using Modified LBP and Optimization Algorithm", The Open Automation and Control Systems Journal, no. 1, (2015), pp. 1916-1921.

[5] G.D. Sun, W. Feng, D.X. Zhao, L.J. Yang, “An automatic fault recognition method for side frame key in TFDS", The Open Mechanical Engineering Journal, vol. 9, no. 1, (2015),pp. 22-27.

[6] N. Li, Z.Z. Wei, Z.P. Cao, "Automatic fault recognition for Brake-Shoe-Key losing of freight train", Optik, vol. 126, no. 23, (2015), pp. 4735-4742.

[7] H J Zhang, P He, X D Yang. "Fault Detection Based on Multi-Scale Local Binary Patterns Operator and Improved Teaching-Learning-Based Optimization Algorithm," Symmetry, vol. 7, no. 4, (2015),pp. 1734-1750.

[8] F.Q. Zhou, R. Zou, Y.F. Qiu, H. Gao, "Automated visual inspection of angle cocks during train operation", Proceedings of Institution of Mechanical Engineers, Part F: Journal of Rail and Rapid Transit, vol. 228, no. 7, (2013), pp. 794-806.

[9] Z.X. Zhu, G.Y. Wang, "A Fast Potential Fault Regions Locating Method Used in Inspecting Freight Cars," Journal of Computers, vol. 9, no. 5, (2014), pp. 1266-1273.

[10] L Y Jiang, "Efficient randomized Hough transform for circle detection using novel probability sampling and feature points," Optik-International Journal for Light and Electron Optics, vol. 123, no. 20, (2012), pp. 1834-1840.

[11] J.X. Zhang, X.L. Shen, H. Wang, J.L. Qiu, B.B. Zhang, "Fast multi-line detection algorithm using randomized Hough transform", Journal of ZheJiang university of technology, vol. 41, no. 3, (2013), pp. 346-350.

[12] Priyanka M, Bidyut B C, “A survey of Hough Transform”, Pattern Recognition, vol. 48, no.3, (2015), pp. 993-1010.

[13] D.X. Meng, E.L. Xie, "Improved algorithm of automobile components circle detection based on Hough transform", Computer Engineering and Applications, vol. 51, no. 11, (2015), pp. 172-174.

[14] L. Yuan, Z.R. Cao, "Improved randomized Hough transform algorithm for circle detection", Journal of Computer Applications, vol. 30, Suppl. 1, (2010), pp.174-176.

[15] F. Zhou, C. Yang, C.G. Wang, B. Q. Wang, "Circle detection and its number identification in complex condition based on random Hough transform", Chinese Journal of Scientific Instrument, vol. 34, no. 3, (2013), pp.622-628. 
[16] W.J. Kang, X.M. Ding, J.W. Cui, L. AO, "Fast straight-line extraction algorithm based on improved Hough Transform", Opto-Electronic Engineering, vol. 34, no. 3, (2007),pp.105-108.

[17] M Wang, "Research of Failure Recognition Algorithm for the Handle Bar and Draw Bar in the Bottom of Freight Car", Huazhong University of Science and Technology, (2012).

[18] Y. Cen, X. Pan, X.J. Gao, X. Liu, "Palmprint recognition using MB-LBP algorithm and HOG algorithm [J/OL]", Application Research of Computers, no. 3, (2017). http://www.cnki.net/kcms/detail/51.1196.TP.20160614.1127.128.html

[19] Ojala T, Pietikainen M, Harwood D. "A comparative study of texture measures with classification based on featured distributions", Pattern recognition, vol. 29,no. 1, (1996), pp. 51-59.

[20] X.F. Yang, Y. Yang. "A Method of Efficient Vehicle Detection Based on HOG-LBP”, Computer Engineering, vol. 40, no. 9, (2014), pp. 210-214.

[21] Y. Huang, C.E. Fan, Q.P. Zhu, H. Zhang, D.X. Deng, "HOG-LBP pedestrian detection", Optics and Precision Engineering, vol. 21, no. 4, (2013), pp. 622-628.

[22] H.M. Ge, L. Chen, J. Liang, Y.Y. Wang, "Parameter Optimizations of Multi-class Support Vector Machine Based on Seeker Optimization Algorithm, International Journal of Control Automation \& Systems", International Journal of Control Automation \& Systems, vol. 9, no. 12, (2016), pp. 235-248.
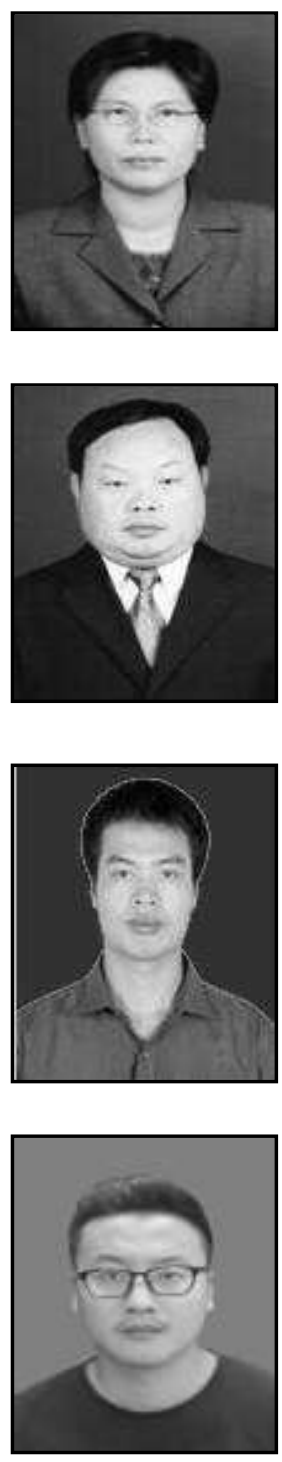

\section{Authors}

Jinmin Zhang received the BS degree in school of automation and electrical engineering from Lanzhou Jiaotong University in 1988. She is currently an associate professor in the College of mechatronic engineering, Lanzhou Jiaotong University, China. Her current research interests include computer vision and pattern recognition.

Siming Wang received the BS degree in school of automation and electrical engineering from Lanzhou Jiaotong University in 1986. He is currently an professor in the College of automation and electrical engineering, Lanzhou Jiaotong University, China. His current research interests include image processing and pattern recognition.

Yingke Feng received the BS degree in school of engineering from south china agricultural University in 2009. $\mathrm{He}$ is currently pursuing the MS degree with College of mechatronic engineering, Lanzhou Jiaotong University. His current research interests include image processing and pattern recognition.

Tianxie Yang received the BS degree in school of engineering from Lanzhou Institute of Technology in 2016.

$\mathrm{He}$ is currently pursuing the MS degree with College of mechatronic engineering, Lanzhou Jiaotong University. His current research interests include image processing and pattern recognition. 
International Journal of Control and Automation Vol.10, No.7 (2017) 